\title{
Paraxial wave function and Gouy phase for a relativistic electron in a uniform magnetic field
}

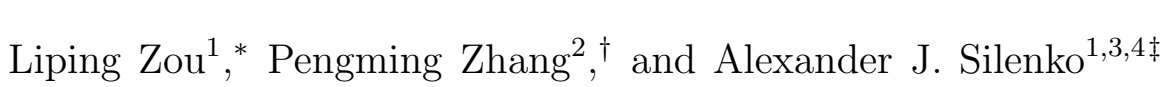 \\ ${ }^{1}$ Institute of Modern Physics, Chinese Academy of Sciences, Lanzhou 730000, China \\ ${ }^{2}$ School of Physics and Astronomy, \\ Sun Yat-sen University, Zhuhai 519082, China \\ ${ }^{3}$ Bogoliubov Laboratory of Theoretical Physics, \\ Joint Institute for Nuclear Research, Dubna 141980, Russia and \\ ${ }^{4}$ Research Institute for Nuclear Problems, \\ Belarusian State University, Minsk 220030, Belarus
}

(Dated: March 11, 2020)

\begin{abstract}
A connection between relativistic quantum mechanics in the Foldy-Wouthuysen representation and the paraxial equations is established for a Dirac particle in external fields. The paraxial form of the Landau eigenfunction for a relativistic electron in a uniform magnetic field is determined. The obtained wave function contains the Gouy phase and significantly approaches to the paraxial wave function for a free electron.
\end{abstract}

\footnotetext{
* zoulp@impcas.ac.cn

$\dagger$ zhangpm5@mail.sysu.edu.cn

‡alsilenko@mail.ru
} 
The prediction [1] and discovery [2] of twisted (vortex) electrons in a free space conditions an importance of a detailed quantum-mechanical description of such particles. For this purpose, the paraxial equation is mostly applied. The approach based on the paraxial equation is widely used in optics for studying twisted and untwisted structured light beams [3-7]. The connection of this approach with traditional approaches of relativistic quantum mechanics (QM) is considered, e.g., in Refs. [8-10].

In contemporary studies of twisted electrons, an important place is occupied by their interactions with a magnetic field [11 24]. A similarity between the wave function for a free twisted electron and the Landau wave function for an electron in a uniform magnetic field is evident and was much discussed (see Refs. [9, 11, 25, 26]). However, approaches used in the two cases substantially differ. The Landau solution [27-29] has been obtained in the framework of nonrelativistic Schrödinger-Pauli QM. The free twisted electron is described by the paraxial equation. The connection between the relativistic QM and the paraxial equation has been analyzed in Refs. [8, 30]. To establish this connection, it is convenient to use the Foldy-Wouthuysen (FW) representation [31]. In this representation, relativistic QM takes the form equivalent to nonrelativistic Schrödinger QM. The Hamiltonian and all operators in the FW representation are even, i.e., block diagonal (diagonal in two spinors). Relations between the operators in this representation are similar to those between the respective classical quantities. The form of quantum-mechanical operators for relativistic particles in external fields is the same as in the nonrelativistic quantum theory. In particular, the operators of the position and momentum are equal to $\boldsymbol{r}$ and $\boldsymbol{p}=-i \hbar \nabla$, respectively [31 37$]$.

The exact relativistic Hamiltonian in the FW representation describing the Dirac electron in the uniform magnetic field $\boldsymbol{B}=B \boldsymbol{e}_{z}$ is defined by [34, 38 40]

$$
i \frac{\partial \Psi_{F W}}{\partial t}=\mathcal{H}_{F W} \Psi_{F W}, \quad \mathcal{H}_{F W}=\beta \sqrt{m^{2}+\boldsymbol{\pi}^{2}-e \boldsymbol{\Sigma} \cdot \boldsymbol{B}}
$$

where $\boldsymbol{\pi}=\boldsymbol{p}-e \boldsymbol{A}$ is the kinetic momentum and $\beta$ and $\boldsymbol{\Sigma}$ are the Dirac matrices. This Hamiltonian acts on the bispinor $\Psi_{F W}=\left(\begin{array}{c}\Phi_{F W} \\ 0\end{array}\right)$. In the present study, we use the system of units $\hbar=1, c=1$. We include $\hbar$ and $c$ explicitly when this inclusion clarifies the problem.

Since eigenfunctions of the FW Hamiltonian (11) are also eigenfunctions of the operator $\boldsymbol{\pi}^{2}$, they are defined by the nonrelativistic Landau solution [38-40]. The eigenfunctions 
are more complicated when the Dirac representation is used [13, 14, 41 44]. Certainly, the energy eigenvalues do not depend on a representation and are given by [13, 14, 34, 38 44]

$$
E=\sqrt{m^{2}+p_{z}^{2}+\left(2 n+1+|\ell|+\ell+2 s_{z}\right)|e| B}
$$

where $n=0,1,2, \ldots$ is the radial quantum number and $\ell$ is an eigenvalue of the orbital angular momentum (OAM) operator projected on the $z$ axis, $\ell=l_{z}=(\boldsymbol{r} \times \boldsymbol{p})_{z}$. In the considered case, $A_{\phi}=\operatorname{Br} / 2, A_{r}=A_{z}=0, e=-|e|$. The relativistic approach (unlike the nonrelativistic one) demonstrates that the Landau levels are not equidistant for any field strength [22]. Amazingly, eigenfunctions (more precisely, upper spinors) of the relativistic FW Hamiltonian are defined by the nonrelativistic Landau solution (see Refs. [38-40]). In the cylindrical coordinates, these eigenfunctions are the Laguerre-Gauss beams:

$$
\begin{gathered}
\Phi_{F W}=\mathcal{A} \exp (i \ell \phi) \exp \left(i p_{z} z\right), \quad \int \Phi_{F W}^{\dagger} \Phi_{F W} r d r d \phi=1, \\
\mathcal{A}=\frac{C_{n \ell}}{w_{m}}\left(\frac{\sqrt{2} r}{w_{m}}\right)^{|\ell|} L_{n}^{|\ell|}\left(\frac{2 r^{2}}{w_{m}^{2}}\right) \exp \left(-\frac{r^{2}}{w_{m}^{2}}\right) \eta \\
C_{n \ell}=\sqrt{\frac{2 n !}{\pi(n+|\ell|) !}}, \quad w_{m}=\frac{2}{\sqrt{|e| B}},
\end{gathered}
$$

where the real function $\mathcal{A}$ defines the amplitude of the beam, and $L_{n}^{|\ell|}$ is the generalized Laguerre polynomial. Since the spin operator in the FW representation, $s=\hbar \Sigma / 2$, commutes with the Hamiltonian (11) and the zero lower spinor of the bispinor $\Psi_{F W}$ is disregarded, the spin function $\eta$ is an eigenfunction of the Pauli operator $\sigma_{z}$ (cf. Ref. [40]):

$$
\sigma_{z} \eta^{+}=\eta^{+}, \quad \sigma_{z} \eta^{-}=-\eta^{-}, \quad \eta^{+}=\left(\begin{array}{l}
1 \\
0
\end{array}\right), \quad \eta^{-}=\left(\begin{array}{l}
0 \\
1
\end{array}\right)
$$

It is important that the solution (3) can be obtained from the exact relativistic wave function in Dirac representation [14] by the use of the connection between the Dirac and FW wave functions found in Ref. [40].

The electron possesses a small anomalous magnetic moment which is not taken into account in Eqs. (2), (3). Due to its existence, a consideration of the spin does not lead to an additional degeneracy of the energy levels. The solution (3) does not contain the Gouy phase.

The use of the FW representation is necessary to connect relativistic quantum-mechanical equations and paraxial ones. The latter equations can be introduced when the paraxial 
approximation $\left|\boldsymbol{p}_{\perp}\right| \ll p$ is satisfied. Operators entering these equations are equivalent to the corresponding operators of Schrödinger QM. Since the FW representation restores the Schrödinger picture of relativistic QM [31 33, 35-37, 45], one needs to use FW Hamiltonians. For the considered problem, the Hamiltonian (11) is exact. In other cases, approximate relativistic FW Hamiltonians can be derived by various methods [34, 35, 46 50].

Similarly to Refs. [8, 10], we can determine a connection between the relativistic quantummechanical equations and paraxial ones for a particle in external fields. In Refs. [8, 10], particles in a free space have been considered. For stationary states, $\mathcal{H}_{F W} \Psi_{F W}=E \Psi_{F W}$. Let us denote $P=\sqrt{E^{2}-m^{2}}=\hbar k$. Squaring Eq. (11) for the upper spinor and applying the paraxial approximation for $p_{z}>0$ results in (cf. Refs. [8, 10])

$$
\begin{gathered}
P^{2}=\boldsymbol{\pi}^{2}-e \boldsymbol{\Sigma} \cdot \boldsymbol{B}=\boldsymbol{\pi}_{\perp}^{2}+p_{z}^{2}-e \boldsymbol{\Sigma} \cdot \boldsymbol{B}, \\
p_{z}=\sqrt{P^{2}-\boldsymbol{\pi}_{\perp}^{2}+e \boldsymbol{\Sigma} \cdot \boldsymbol{B}} \approx P-\frac{\boldsymbol{\pi}_{\perp}^{2}-e \boldsymbol{\Sigma} \cdot \boldsymbol{B}}{2 P} .
\end{gathered}
$$

This transformation leads to the following equation:

$$
\left(\boldsymbol{\pi}_{\perp}^{2}-e \boldsymbol{\Sigma} \cdot \boldsymbol{B}+2 P p_{z}\right) \Phi_{F W}=2 P^{2} \Phi_{F W}
$$

An equivalent form of this equation reads

$$
\begin{aligned}
\left(\boldsymbol{\pi}_{\perp}^{2}-e \boldsymbol{\Sigma} \cdot \boldsymbol{B}-2 i k \frac{\partial}{\partial z}\right) \Phi_{F W} & =2 k^{2} \Phi_{F W}, \quad \boldsymbol{\pi}_{\perp}^{2}=-\nabla_{\perp}^{2}+i e B \frac{\partial}{\partial \phi}+\frac{e^{2} B^{2} r^{2}}{4}, \\
\nabla_{\perp}^{2} & =\frac{\partial^{2}}{\partial r^{2}}+\frac{1}{r} \frac{\partial}{\partial r}+\frac{1}{r^{2}} \frac{\partial^{2}}{\partial \phi^{2}} .
\end{aligned}
$$

Equation (6) is an approximate form of the general equation (11) when the paraxial approximation is satisfied. The substitution $\Phi_{F W}=\exp (i k z) \Psi$ brings the corresponding paraxial equation

$$
\left(\nabla_{\perp}^{2}-i e B \frac{\partial}{\partial \phi}-\frac{e^{2} B^{2} r^{2}}{4}+2 e s_{z} B+2 i k \frac{\partial}{\partial z}\right) \Psi=0 .
$$

This substitution shifts the squared particle momentum and is equivalent to a shift of the zero energy level in Schrödinger QM. Within the paraxial approximation, Eq. (7) properly describes electrons of arbitrary energies in a uniform magnetic field. Therefore, the use of the FW transformation establishes a connection between relativistic QM and the respective relativistic paraxial equation. We underline the difference between $\Phi_{F W}$ and $\Psi$.

The Landau solution defines the eigenvalues of the operator describing the transversal motion:

$$
\left(\nabla_{\perp}^{2}-i e B \frac{\partial}{\partial \phi}-\frac{e^{2} B^{2} r^{2}}{4}+2 e s_{z} B\right) \Phi_{F W}=-\left(2 n+1+|\ell|+\ell+2 s_{z}\right)|e| B \Phi_{F W} .
$$


The same equation can be written for the paraxial wave function $\Psi$. This equation allows us to determine the Gouy phase.

The Landau wave function contains the exponential factor $\exp \left[i\left(p_{z} / \hbar\right) z\right]$. Taking into account the connection between $\Phi_{F W}$ and $\Psi$, we obtain that the latter wave function is proportional to the exponential factor

$$
\exp \left(i \frac{p_{z}}{\hbar} z\right) \exp (-i k z)=\exp \left(-i \frac{P-p_{z}}{\hbar} z\right)
$$

Equations (3), (44), and (8) result in the following form of the paraxial wave function:

$$
\begin{gathered}
\Psi=\mathcal{A} \exp (i \ell \phi) \exp [-i \zeta(z)], \quad \int \Psi^{\dagger} \Psi r d r d \phi=1 \\
\zeta=\left(2 n+1+|\ell|+\ell+2 s_{z}\right) \frac{|e| B}{2 k} z=\left(2 n+1+|\ell|+\ell+2 s_{z}\right) \frac{2 z}{k w_{m}^{2}}
\end{gathered}
$$

where $\zeta$ is the Gouy phase. Evidently, this wave function satisfies the paraxial equation (7).

Equation (91) shows that the wave eigenfunction of the relativistic electron in the uniform magnetic field acquires the Gouy phase $\zeta$ after the transition to the paraxial equation. This property increases the similarity between the wave eigenfunctions of the relativistic Landau electron in the uniform magnetic field and the twisted electron in a free space. In the latter case, the eigenfunction reads [ $\left[\begin{array}{ll}9 & 11\end{array}\right]$

$$
\begin{gathered}
\Psi=\mathbb{A} \exp (i \ell \phi) \exp \left[i \frac{k r^{2}}{2 R(z)}\right] \exp [-i \zeta(z)] \\
\mathbb{A}=\frac{C_{n \ell}}{w(z)}\left(\frac{\sqrt{2} r}{w(z)}\right)^{|\ell|} L_{n}^{|\ell|}\left(\frac{2 r^{2}}{w^{2}(z)}\right) \exp \left(-\frac{r^{2}}{w^{2}(z)}\right) \eta, \\
C_{n \ell}=\sqrt{\frac{2 n !}{\pi(n+|\ell|) !}}, \quad w(z)=w_{0} \sqrt{1+\frac{z^{2}}{z_{R}^{2}}}, \quad R(z)=z+\frac{z_{R}^{2}}{z}, \\
\zeta(z)=(2 n+|\ell|+1) \arctan \left(\frac{z}{z_{R}}\right), \quad z_{R}=\frac{k w_{0}^{2}}{2},
\end{gathered}
$$

where $w_{0}$ is the minimum beam width, $R(z)$ is the radius of curvature of the wavefront, and $z_{R}$ is the Rayleigh diffraction length.

An analysis of Eqs. (3), (9), and (10) shows the substantial similarity between the paraxial wave functions of relativistic Dirac electrons in a uniform magnetic field and a free space. The former case (Landau solution) characterizes the wave with the infinite radius of curvature of the wavefront, $R(z) \rightarrow \infty$. In this case, $z_{R} \rightarrow \infty, \arctan \left(z / z_{R}\right) \approx z / z_{R}$, and the paraxial wave functions becomes equivalent provided that $w_{0}=w_{m}$. 
It can be similarly obtained that the paraxial wave function of relativistic positrons $(e=|e|)$ in a uniform magnetic field has the form (9) where the Gouy phase differs by signs:

$$
\zeta=\left(2 n+1+|\ell|-\ell-2 s_{z}\right) \frac{|e| B}{2 k} z=\left(2 n+1+|\ell|-\ell-2 s_{z}\right) \frac{2 z}{k w_{m}^{2}} .
$$

The Landau solution describes a motion of a charged particle in a uniform magnetic field. This motion is governed by the Lorentz force and a direction of a particle rotation is definite. A simple analysis shows that physically correct solutions of the related quantum-mechanical equations correspond to $\ell \geq 0$ for electrons and $\ell \leq 0$ for positrons.

The obtained connection between relativistic QM in the FW representation and the paraxial equations can be applied to a wide class of problems connected with relativistic electron (positron, muon) beams in different external fields [51 57] (e.g., crossed magnetic and electric fields).

In summary, we have presented the first attempt to establish a connection between relativistic QM in the FW representation and the paraxial equations for a Dirac particle in external fields. For a relativistic electron in a uniform magnetic field, the paraxial form of the Landau eigenfunction contains the Gouy phase and amazingly approaches to the paraxial wave function for a free electron. The Gouy phase does not enter the standard quantummechanical solutions in the Dirac and FW representations. We have demonstrated for the first time that it appears as a result of a transition from QM in the FW representation (or Schrödinger-Pauli QM) to the paraxial quantum-mechanical equation.

\section{ACKNOWLEDGMENTS}

This work was supported by the Belarusian Republican Foundation for Fundamental Research (Grant No. \$18D-002), by the National Natural Science Foundation of China (Grants No. 11575254 and No. 11805242), and by the National Key Research and Development Program of China (No. 2016YFE0130800). A. J. S. also acknowledges hospitality and support by the Institute of Modern Physics of the Chinese Academy of Sciences. The authors are 
grateful to I. P. Ivanov for helpful exchanges.

[1] K. Bliokh, Y. Bliokh, S. Savel'ev, and F. Nori, Semiclassical Dynamics of Electron Wave Packet States with Phase Vortices, Phys. Rev. Lett. 99, 190404 (2007).

[2] M. Uchida, A. Tonomura, Generation of electron beams carrying orbital angular momentum, Nature (London) 464, 737 (2010); J. Verbeeck, H. Tian, P. Schattschneider, Production and application of electron vortex beams, Nature (London) 467, 301 (2010).

[3] A. E. Siegman, Lasers (University Science Books, Sausalito, 1986).

[4] L. Allen, M. W. Beijersbergen, R. J. C. Spreeuw, J. P. Woerdman, Orbital angular momentum of light and the transformation of Laguerre-Gaussian laser modes, Phys. Rev. A 45, 8185 (1992).

[5] A. Bekshaev, M. Soskin, M. Vasnetsov, Paraxial Light Beams with Angular Momentum (Nova Science Publishers, New York, 2008).

[6] S. Franke-Arnold, L. Allen, M. J. Padgett, Advances in optical angular momentum, Laser Photonics Rev. 2, 299 (2008).

[7] S. M. Barnett, M. Babiker and M. J. Padgett, Optical orbital angular momentum, Phil. Trans. R. Soc. A 375, 20150444 (2017).

[8] S. M. Barnett, Optical Dirac equation, New J. Phys. 16, 093008 (2014).

[9] K. Y. Bliokh, I. P. Ivanov, G. Guzzinati, L. Clark, R. Van Boxem, A. Béché, R. Juchtmans, M. A. Alonso, P. Schattschneider, F. Nori, and J. Verbeeck, Theory and applications of freeelectron vortex states, Phys. Rep. 690, 1 (2017).

[10] A. J. Silenko, Pengming Zhang, and Liping Zou, Relativistic quantum-mechanical description of twisted paraxial electron and photon beams, Phys. Rev. A 100, 030101(R) (2019).

[11] K. Y. Bliokh, P. Schattschneider, J. Verbeeck, F. Nori, Electron vortex beams in a magnetic field: A new twist on Landau levels and Aharonov-Bohm states, Phys. Rev. X 2, 041011 (2012).

[12] G. M. Gallatin, B. McMorran, Propagation of vortex electron wave functions in a magnetic field, Phys. Rev. A 86, 012701 (2012); C. R. Greenshields, R. L. Stamps, S. Franke-Arnold, S. M. Barnett, Is the angular momentum of an electron conserved in a uniform magnetic field? Phys. Rev. Lett. 113240404 (2014); D. Chowdhury, B. Basu, and P. Bandyopadhyay, 
Electron vortex beams in a magnetic field and spin filter, Phys. Rev. A 91, 033812 (2015).

[13] K. van Kruining, A. G. Hayrapetyan, and J. B. Götte, Nonuniform currents and spins of relativistic electron vortices in a magnetic field, Phys. Rev. Lett. 119, 030401 (2017).

[14] A. Rajabi and J. Berakdar, Relativistic electron vortex beams in a constant magnetic field, Phys. Rev. A 95, 063812 (2017).

[15] C. Greenshields, R. L. Stamps, and S. Franke-Arnold, Vacuum Faraday effect for electrons, New J. Phys. 14, 103040 (2012).

[16] Y. D. Han, T. Choi, Classical understanding of electron vortex beams in a uniform magnetic field, Phys. Lett. A 381, 1335 (2017).

[17] M. Babiker, J. Yuan, and V. E. Lembessis, Electron vortex beams subject to static magnetic fields, Phys. Rev. A 91, 013806 (2015); J. Qiu, C. Ren, and Z. Zhang, Precisely measuring the orbital angular momentum of beams via weak measurement, Phys. Rev. A 93063841 (2016).

[18] J. Rusz, S. Bhowmick, M. Eriksson, N. Karlsson, Scattering of electron vortex beams on a magnetic crystal: Towards atomic-resolution magnetic measurements, Phys. Rev. B 89, 134428 (2014).

[19] A. Edström, A. Lubk, J. Rusz, Elastic scattering of electron vortex beams in magnetic matter, Phys. Rev. Lett. 116, 127203 (2016).

[20] V. Grillo, T. R. Harvey, F. Venturi, J. S. Pierce, R. Balboni, F. Bouchard, G. C. Gazzadi, S. Frabboni, A. H. Tavabi, Z. Li, R. E. Dunin-Borkowski, R. W. Boyd, B. J. McMorran, E. Karimi, Observation of nanoscale magnetic fields using twisted electron beams, Nat. Commun. 8, 689 (2017).

[21] A. J. Silenko, Pengming Zhang and Liping Zou, Manipulating Twisted Electron Beams, Phys. Rev. Lett. 119, 243903 (2017).

[22] A. J. Silenko, Pengming Zhang and Liping Zou, Relativistic quantum dynamics of twisted electron beams in arbitrary electric and magnetic fields, Phys. Rev. Lett. 121, 043202 (2018).

[23] A. J. Silenko, Pengming Zhang, and Liping Zou, Electric Quadrupole Moment and the Tensor Magnetic Polarizability of Twisted Electrons and a Potential for their Measurements, Phys. Rev. Lett. 122, 063201 (2019).

[24] A. J. Silenko and O. V. Teryaev, Siberian Snake-Like Behavior for an Orbital Polarization of a Beam of Twisted (Vortex) Electrons, Phys. Part. Nucl. Lett. 16, 77 (2019).

[25] S. M. Lloyd, M. Babiker, G. Thirunavukkarasu, and J. Yuan, Electron vortices: Beams with 
orbital angular momentum, Rev. Mod. Phys. 89, 035004 (2017).

[26] H. Larocque, I. Kaminer, V. Grillo, G. Leuchs, M. J. Padgett, R. W. Boyd, M. Segev, E. Karimi, 'Twisted' electrons, Contemp. Phys. 59, 126 (2018).

[27] V. Fock, Bemerkung zur quantelung des harmonischen oszillators im magnetfeld, Z. Phys. 47, 446 (1928).

[28] L. D. Landau, Diamagnetismus der Metalle, Z. Phys. 64, 629 (1930).

[29] L. D. Landau, E.M. Lifshitz, Quantum Mechanics. Non-Relativistic Theory, 3rd ed. (Pergamon Press, Oxford, 1977), p. 458.

[30] A. J. Silenko, Pengming Zhang, and Liping Zou, Relativistic quantum-mechanical description of twisted paraxial electron and photon beams, Phys. Rev. A 100, 030101(R) (2019).

[31] L. L. Foldy, S. A. Wouthuysen, On the Dirac Theory of Spin 1/2 Particles and Its NonRelativistic Limit, Phys. Rev. 78, 29 (1950).

[32] J. P. Costella and B. H. J. McKellar, The Foldy-Wouthuysen transformation, Am. J. Phys. 63, 1119 (1995).

[33] E. de Vries, Foldy-Wouthuysen Transformations and Related Problems, Fortschr. Phys. 18, 149 (1970).

[34] A. J. Silenko, Foldy-Wouthuysen transformation for relativistic particles in external fields, J. Math. Phys. 44, 2952 (2003).

[35] A. J. Silenko, General properties of the Foldy-Wouthuysen transformation and applicability of the corrected original Foldy-Wouthuysen method, Phys. Rev. A 93, 022108 (2016).

[36] A. J. Silenko, Pengming Zhang, and Liping Zou, Silenko, Zhang, and Zou Reply, Phys. Rev. Lett. 122, 159302 (2019).

[37] A. J. Silenko, Pengming Zhang, and Liping Zou, Position and spin in relativistic quantum mechanics (to be published).

[38] K. M. Case, Some Generalizations of the Foldy-Wouthuysen Transformation, Phys. Rev. 95, 1323 (1954).

[39] W. Tsai, Energy eigenvalues for charged particles in a homogeneous magnetic field - an application of the Foldy-Wouthuysen transformation, Phys. Rev. D 7, 1945 (1973).

[40] A. J. Silenko, Connection between wave functions in the Dirac and Foldy-Wouthuysen representations, Phys. Part. Nucl. Lett. 5, 501 (2008).

[41] I. M. Ternov, V. G. Bagrov, and V. Ch. Zhukovsky, Synchrotron radiation of electron with 
vacuum magnetic moment, Mosc. Univ. Phys. Bull. 21, 21 (1966).

[42] A. A. Sokolov and I. M. Ternov, Radiation from relativistic electrons, 2nd ed. (AIP, New York, 1986).

[43] R. F. O'Connell, Motion of a relativistic electron with an anomalous magnetic moment in a constant magnetic field, Phys. Lett. A 27, 391 (1968).

[44] V. Canuto and H. Y. Chiu, Quantum Theory of an Electron Gas in Intense Magnetic Field, Phys. Rev. 173, 1210 (1968).

[45] A. J. Silenko, Classical limit of relativistic quantum mechanical equations in the FoldyWouthuysen representation, Phys. Part. Nucl. Lett. 10, 91 (2013).

[46] A. J. Silenko, Foldy-Wouthyusen transformation and semiclassical limit for relativistic particles in strong external fields, Phys. Rev. A 77, 012116 (2008).

[47] A. J. Silenko, Comparative analysis of direct and "step-by-step" Foldy-Wouthuysen transformation methods, Teor. Mat. Fiz. 176, 189 (2013) [Theor. Math. Phys. 176, 987 (2013)].

[48] A. J. Silenko, General method of the relativistic Foldy-Wouthuysen transformation and proof of validity of the Foldy-Wouthuysen Hamiltonian, Phys. Rev. A 91, 022103 (2015).

[49] Dah-Wei Chiou and Tsung-Wei Chen, Exact Foldy-Wouthuysen transformation of the DiracPauli Hamiltonian in the weak-field limit by the method of direct perturbation theory, Phys. Rev. A 94, 052116 (2016).

[50] A. J. Silenko, Exact form of the exponential Foldy-Wouthuysen transformation operator for an arbitrary-spin particle, Phys. Rev. A 94, 032104 (2016).

[51] V. B. Berestetskii, E. M. Lifshitz, L. P. Pitaevskii, Quantum Electrodynamics, 2nd ed. (Pergamon Press, Oxford, U.K., 1982).

[52] A. V. Glushkov, Relativistic quantum theory. Quantum mechanics of atomic systems (Odessa: Astroprint, 2008).

[53] W. Ihra, F. Mota-Furtado, and P. F. O'Mahony, Ionization of atoms in parallel electric and magnetic fields: The role of classical phase space, Phys. Rev. A 58, 3884 (1998).

[54] J. Rao, D. Delande and K. T. Taylor, Quantum manifestations of scattering orbits in the scaled spectrum of a non-hydrogenic atom in crossed fields, J. Phys. B: At. Mol. Opt. Phys. 35, L1 (2002).

[55] G. A. Gottwald and I. Melbourne, Testing for chaos in deterministic systems with noise, Physica D 212, 100 (2005). 
[56] A. V. Glushkov, Multiphoton Spectroscopy of Atoms and Nuclei in a Laser Field: Relativistic Energy Approach and Radiation Atomic Lines Moments Method, Advances in Quantum Chem. 78, 253 (2019).

[57] J. Chávez-Carlos, B. López-del-Carpio, M. A. Bastarrachea-Magnani, P. Stránský, S. LermaHernández, L. F. Santos, J. G. Hirsch, Quantum and Classical Lyapunov Exponents in AtomField Interaction Systems, Phys. Rev. Lett. 122, 024101 (2019). 